\title{
Semiconductor wafer defect detection using digital holography
}

\author{
Mark A. Schulze, Martin A. Hunt, Edgar Voelkl, Joel D. Hickson, William Usry, \\ Randall G. Smith, Robert Bryant, C. E. (Tommy) Thomas Jr. \\ nLine Corporation, 4150 Freidrich Lane, Suite A, Austin, Texas 78744
}

\begin{abstract}
Defect inspection metrology is an integral part of the yield ramp and process monitoring phases of semiconductor manufacturing. High aspect ratio structures have been identified in the ITRS as critical structures where there are no known manufacturable solutions for defect detection. We present case studies of a new inspection technology based on digital holography that addresses this need. Digital holography records the amplitude and phase of the wavefront from the target object directly to a single image acquired by a CCD camera. Using deep ultraviolet laser illumination, digital holography is capable of resolving phase differences corresponding to height differences as small as several nanometers. Thus, the technology is well suited to the task of finding defects on semiconductor wafers. We present a study of several defect detection benchmark wafers, and compare the results of digital holographic inspection to other wafer inspection technologies. Specifically, digital holography allows improved defect detection on high aspect ratio features, such as improperly etched contacts. In addition, the phase information provided by digital holography allows us to visualize the topology of defects, and even generate three-dimensional images of the wafer surface comparable to scanning electron microscope (SEM) images. These results demonstrate the unique defect detection capabilities of digital holography.
\end{abstract}

Keywords: digital holography, semiconductor wafer defects, semiconductor metrology, high aspect ratio inspection (HARI)

\section{INTRODUCTION}

For the past few years, the International Technology Roadmap for Semiconductors (ITRS) [1] has highlighted the increasing need for technologies that address key defect detection and characterization requirements. Specifically, the need to inspect high aspect ratio structures and differentiate between killer and non-killer defects have been rated as difficult challenges without known manufacturable solutions. The roadmap has called for specific research into these areas in order to achieve the required sensitivity and reasonable cost of ownership.

Currently there are three main technologies in use for defect detection: dark field inspection, bright field inspection, and scanning electron microscopy. The physics of all of three of these technologies will limit them in at least one of the requirements necessary to reach the goals of the current technology roadmap. nLine Corporation has been developing a new defect inspection technology based on novel sensor physics $[4,5]$ that has the potential to overcome the current industry limitations. In addition, the detection technology provides direct information about the topology and material characteristics than can be used to aid the differentiation of defect severity. Thomas, et al. reported on the fundamentals of a novel digital holography implementation and its application in the semiconductor inspection and review processes [6]. The first production ready version of an inspection tool based on this technology, named Fathom ${ }^{\mathrm{TM}}$, has been in the characterization and evaluation process over the past 9 months. In this paper we report on a few examples that demonstrate the capabilities of the Fathom tool.

The paper will present a brief overview of the digital holography technology incorporated in the Fathom tool and how it is used in for semiconductor defect detection. Unique capabilities will be highlighted and examples presented that show the added dimension available using digital holography. Following the technology overview, several example defect detection cases will be given to demonstrate the technology.

Copyright 2003 Society of Photo-Optical Instrumentation Engineers.

This paper will be published in Process and Materials Characterization and Diagnostics in IC Manufacturing II and is made available as an electronic preprint with permission of SPIE. One print or electronic copy may be made for personal use only. Systematic or multiple reproduction, distribution to multiple locations via electronic or other means, duplication of any material in this paper for a fee or for commercial purposes, or modification of the content of the paper are prohibited. 


\section{TECHNOLOGY OVERVIEW}

The nLine Fathom tool uses a digital implementation of modern heterodyne holography as first described by Leith and Upatnieks [2,3]. Using a very small mixing angle between the object and reference waves, the resulting holographic image exhibits fringes at a high spatial frequency that "carry" the phase information. Using appropriate algorithms, the complete object wave (amplitude and phase) can be reconstructed from the digitally recorded hologram. A schematic diagram of the basic arrangement of the Fathom tool is shown in Figure 1 below [5]. The system uses a Mach-Zehnder interferometer arrangement with illumination through the lens. Because of the small feature sizes used on semiconductor wafers, the wavelength of the illumination must be as small as practical in order to penetrate the structures. The current version of the Fathom tool uses deep ultraviolet (DUV) laser illumination with a wavelength of $266 \mathrm{~nm}$.

A portion of a hologram taken on the Fathom tool is show in Figure 2 below. The interference fringes that carry the phase information of the wave emanating from the wafer surface are clearly visible. Further details about the technology incorporated into the Fathom tool are described in Thomas et al. [6, 7].

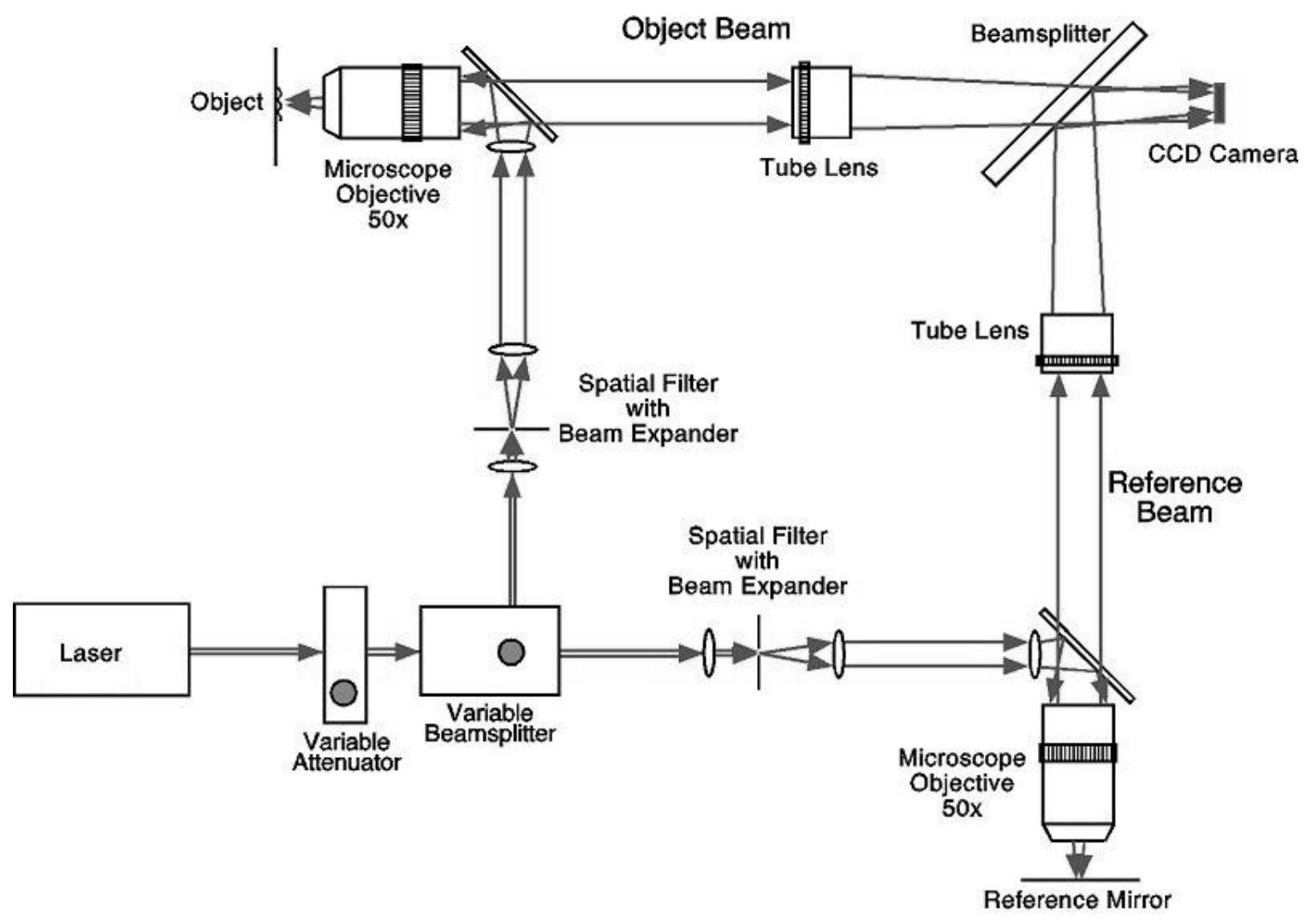

Figure 1. Diagram of a digital holography system using a Mach-Zehnder configuration and through-thelens illumination, similar to the nLine Fathom tool. 


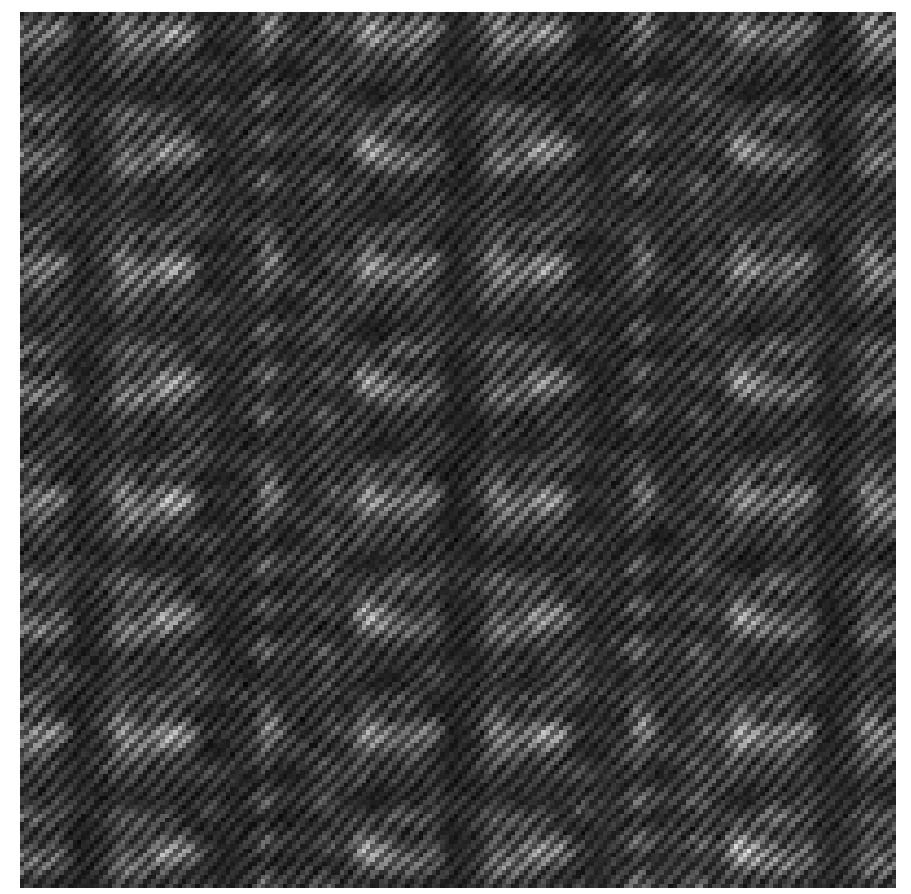

Figure 2. Portion of a digitally recorded off-axis hologram, showing interference fringes.

\section{IMAGE RECONSTRUCTION AND DEFECT DETECTION}

\subsection{Image reconstruction}

A series of computations are required to reconstruct the complete wavefront returning from the wafer surface from the off-axis hologram and its interference fringes. The first step is to compute the Fourier transform of the hologram. For a patterned wafer surface, the amplitude of the Fourier transform (scaled logarithmically) will resemble Figure 3 below. The interference fringes create a carrier (heterodyne) frequency some distance away from the center (autocorrelation peak) of the transform. The information contained in the sideband around this carrier frequency is the (resolution-limited) complex wavefront information. By extracting one of the two sidebands from the Fourier transform, applying a digital lowpass filter to remove any unwanted contribution from the autocorrelation band or possible aliased frequencies (see Figure 4), and computing the inverse Fourier transform, the complex wavefront from the target arm is reproduced in digital form [5-9]. This digital reproduction is, of course, subject to the distortions introduced to it by the optical imaging system and also by computational and quantization error. With a well-designed (diffraction-limited) optical system and the use of appropriate bit lengths on the acquired and processed data, these distortions are minimal.

The reconstructed complex wavefront is usually visualized as two separate images, one representing the amplitude of the complex wave and the other representing the phase. This is shown in Figure 5 below. In this case, the phase image has been corrected using the reconstructed image of a flat surface in the object arm $[7,8]$. This technique corrects for any curvature present in the object wave that is not caused by the object itself. While this step is not essential for defect detection, it does improve the visual appearance of the phase images because it removes distracting phase wraps (where the phase jumps suddenly between $-\pi$ radians to $+\pi$ radians) from the image. Note that because phase changes are computed in a manner that makes them insensitive to these sudden jumps [11], phase wraps are not an impediment to accurate defect detection. 


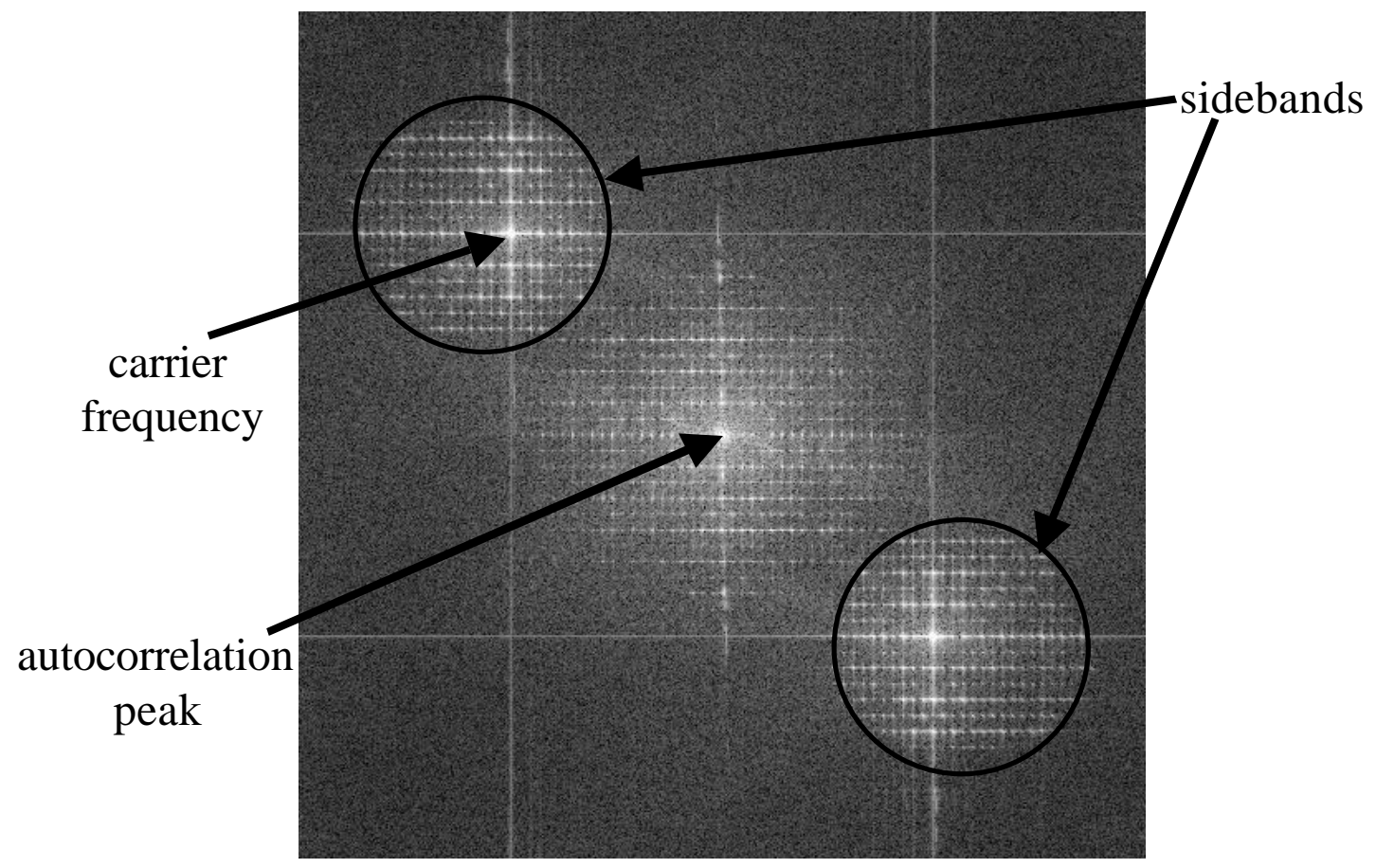

Figure 3. Fourier transform of a hologram of a patterned wafer surface acquired on the Fathom tool. (Logarithmically scaled amplitude of transform is displayed.)

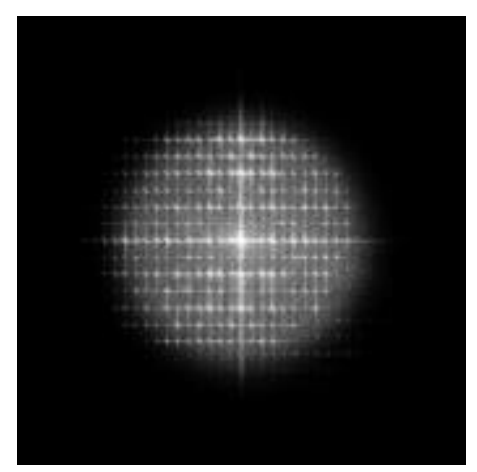

Figure 4. Lowpass filtered sideband taken from the transform shown in Figure 3. 

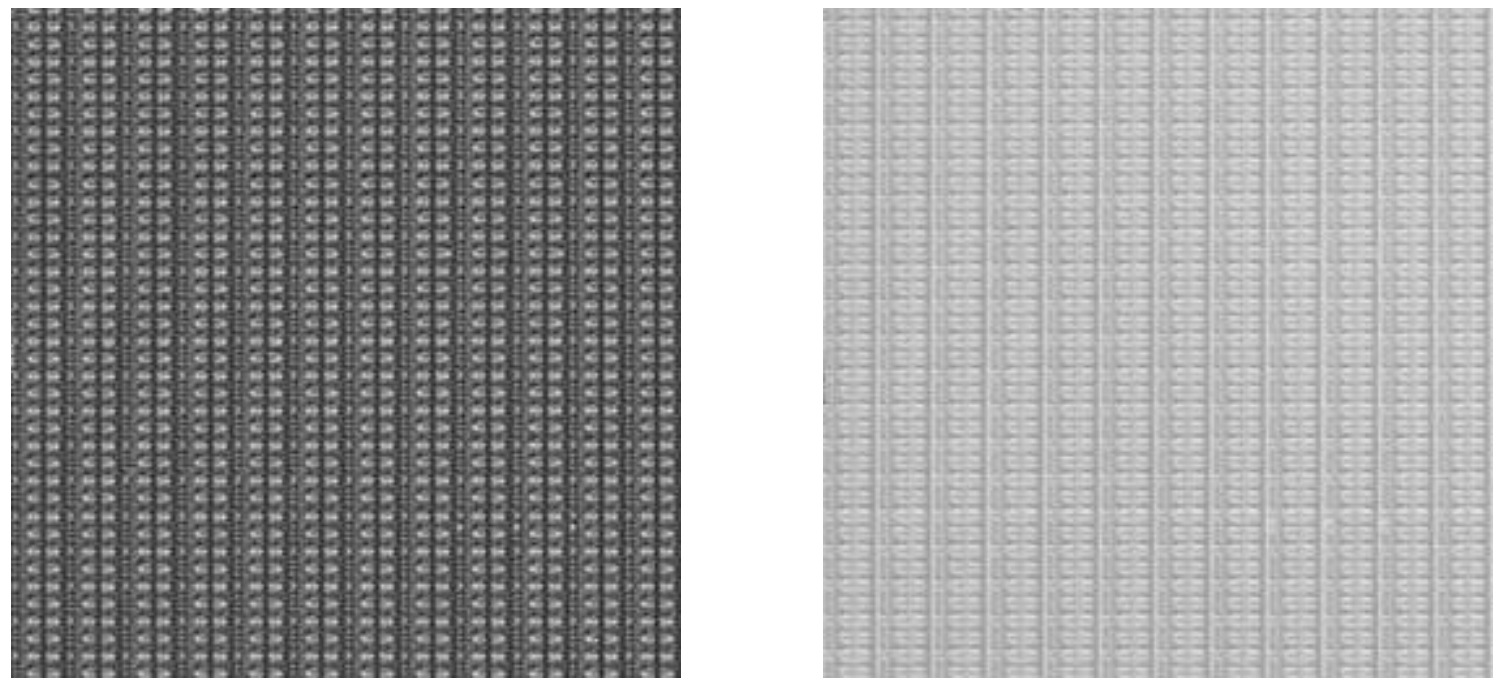

Figure 5. Amplitude (left) and phase (right) images computed from the complex image reconstructed from the example shown in Figure 2, Figure 3 and Figure 4.

\subsection{Defect detection}

Defect detection is performed by directly comparing the two complex wavefronts taken from corresponding fields of view from adjacent die on the wafer. To isolate which field of view a detected defect is in, two comparisons are made for each field of view, and the defect is ascribed to that field only if it appears in both difference images. Difference images can be computed as either amplitude or phase differences (see Figure 12 for examples) or as a composite difference (see Figure 11 for an example).

In order to compare two images from different die, the pixel values must be aligned in the $x$ and $y$ directions (spatial registration) and matched in terms of their overall intensity and phase offset (normalization). Spatial registration of the complex images is performed via an automatic registration algorithm described previously [10]. Normalization consists of computing statistics of the average amplitude and phase of the two images to be compared, and adjusting the average amplitude and phase of one of the images to match the statistics of the other image. One way of computing the average amplitude and phase of the images is simply to sum the complex pixel values at every point and divide by the number of pixels. Alternatively, the average of the amplitude values at each pixel can be computed, and the phase average determined by averaging the complex values of unit-normalized pixel values. Typically, the averages found by these two methods are very close, but not identical. These and other methods of extracting statistics from phase information are found in Fisher [11].

\section{EXAMPLES}

\subsection{Visualization of surface variations via phase information}

The phase information derived from the reconstructed complex images on the Fathom tool changes with surface height variations and index of refraction changes on the wafer. We have found in many instances that phase images of some wafer structures have a similar visual appearance to images taken on a scanning electron microscope (SEM). In Figure 6 below, images taken with a SEM of several different types of defects on an intentional defect array are compared to a phase image taken with the Fathom tool. While the resolution of the Fathom tool is much lower because it is an optical tool, the defects (which are approximately $250 \mathrm{~nm}$ and $125 \mathrm{~nm}$ ) are clearly seen. 


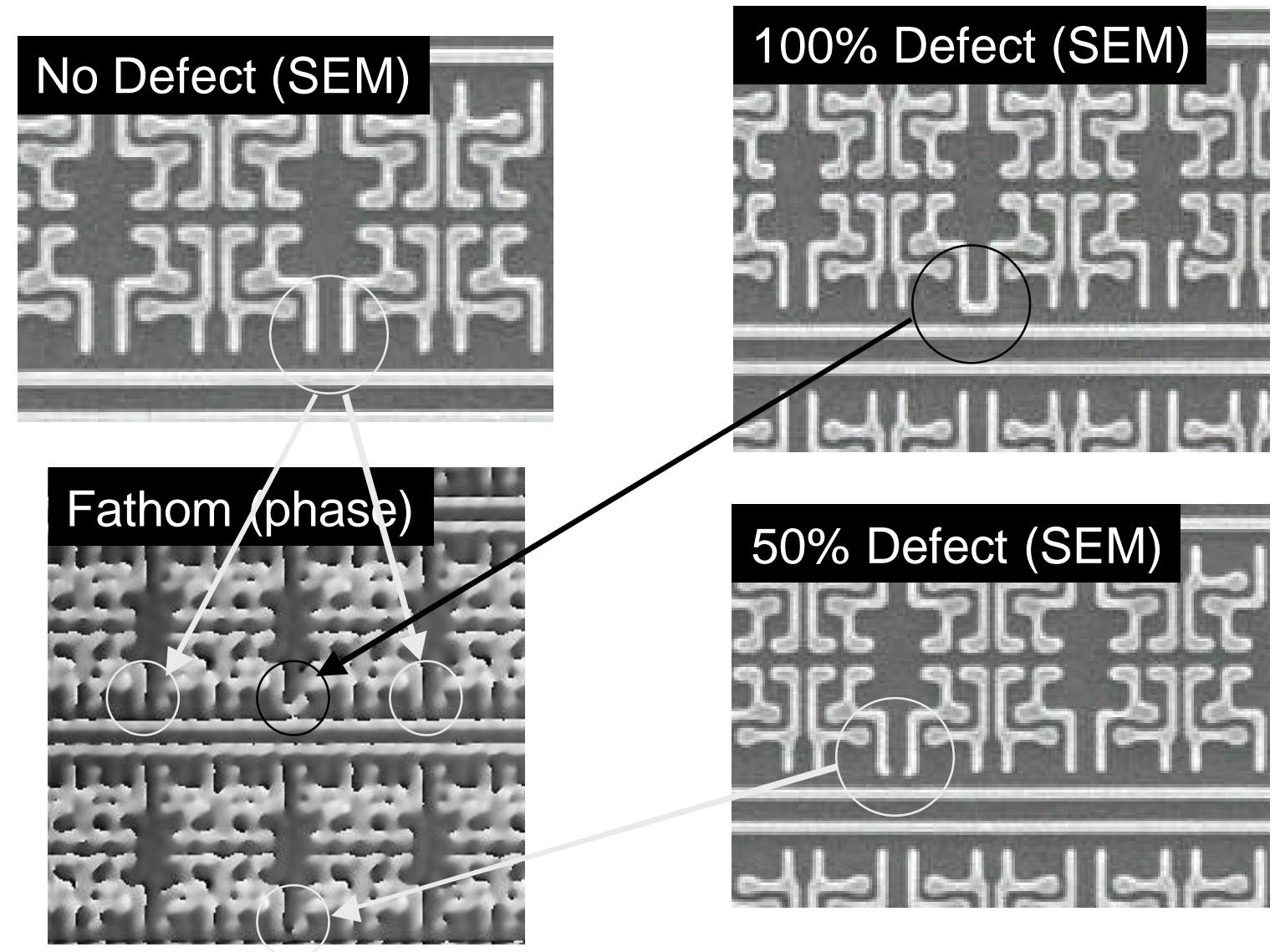

Figure 6. Comparison of Fathom phase image vs. scanning electron microscope images.

(Courtesy International SEMATECH)

\subsection{Visualization and detection of under-etched high aspect ratio contacts}

International SEMATECH has prepared and made available a set of High Aspect Ratio Inspection (HARI) Intentional Defective Contact wafers. The wafers have silicon dioxide thicknesses ranging from $250 \mathrm{~nm}$ to $1500 \mathrm{~nm}$. Contact holes of $220 \mathrm{~nm}$ diameter were etched in four $1 \mathrm{~mm}$ square arrays at a pitch of $660 \mathrm{~nm}$. The resulting aspect ratios range from 1.2:1 to 6.8:1. A significant point is that the contact diameter (220 $\mathrm{nm})$ is smaller than the wavelength of the Fathom's deep UV laser $(266 \mathrm{~nm})$. Five programmed defects are located at positions denoted by arrows etched in the wafer. These defects are incompletely etched contact holes, containing $80 \mathrm{~nm}$ of residual oxide, in the following configurations: $1,1 \times 2,2 \times 2,3 \times 3$, and $4 \times 4$ adjacent defective contacts. Figure 7 below shows a SEM cross section of a single defective contact from a 4.5:1 aspect ratio wafer. The incompletely etched contact has $80 \mathrm{~nm}$ of residual silicon dioxide at the bottom of a single contact, which is $990 \mathrm{~nm}$ deep. 


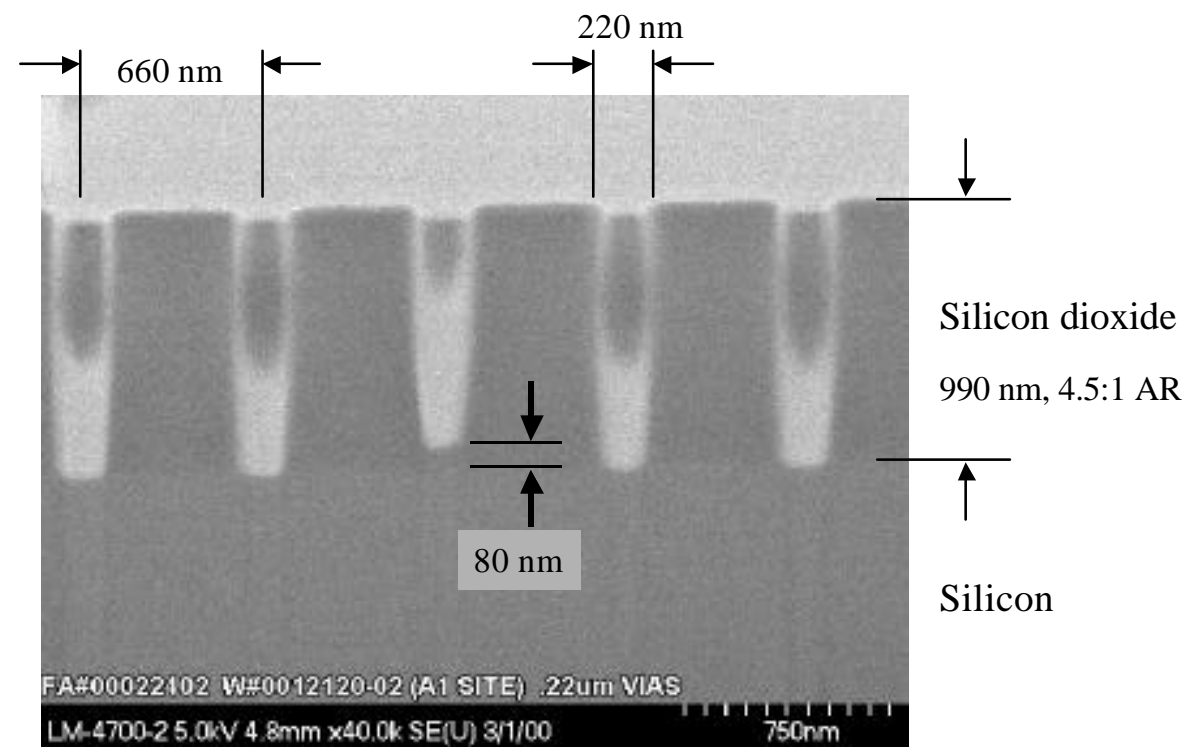

Figure 7. Cross section of high aspect ratio contact defect on test wafer.

(Courtesy International SEMATECH)

The high aspect ratio under-etched contact is easily seen on the Fathom tool using both phase and amplitude imagery. Figure 8 shows a phase image of a single defective contact reconstructed from a hologram taken on the Fathom tool. The images shown were acquired at high magnification (that is, in "review mode"), and the defect is clearly visible. The defective contact stands out in the phase image from the normal contacts due to the contact depth difference as well as the refractive index difference between silicon dioxide and silicon. The defect signal return is so large that a subtraction reference image is not necessary even though the contacts are not completely resolved.
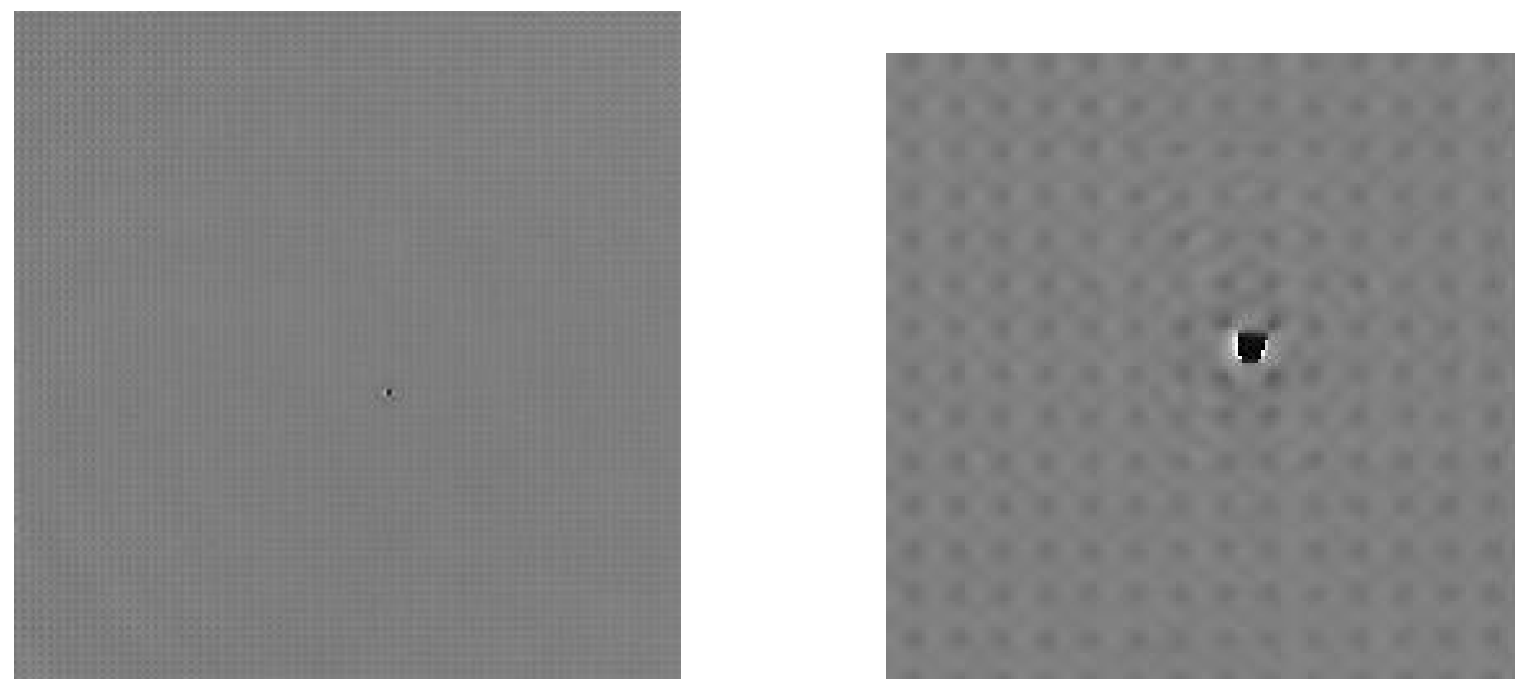

Figure 8. Phase image of a single under-etched contact taken on the Fathom tool at high magnification. (Left) Entire field of view. (Right) Portion of phase image enlarged to show detail.

The defective contact is also visible in the reconstructed amplitude image, as shown in Figure 9 below. The "ringing" around the defective contact is an expected consequence of the defect profile and diffraction of the coherent illumination source; this effect is described in more detail in Thomas, et al. [7]. 

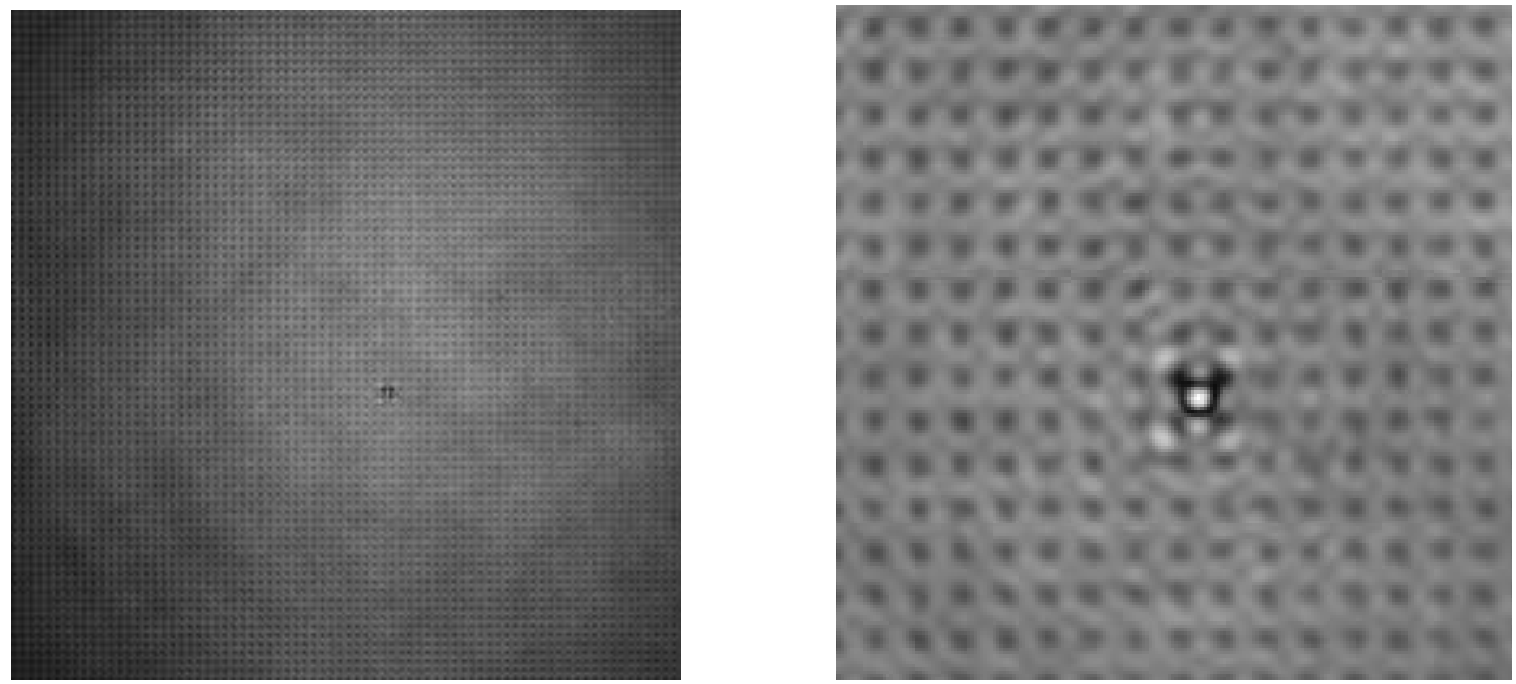

Figure 9. Amplitude image of a single under-etched contact taken on the Fathom tool at high magnification. (Left) Entire field of view. (Right) Portion of amplitude image enlarged to show detail.

At lower magnification ("inspection mode"), the under-etched contacts are not resolved well enough to be visible in either the reconstructed phase or amplitude images, but the difference in signal is still large enough for the defects to be easily detected. Figure 10 below shows the reconstructed phase image of a low magnification field of view taken at a location on the test wafer with both a single under-etched contact and a 1x2 array of under-etched contacts. The defects are not visible in the image. Figure 11 shows the difference image for this field of view compared to the corresponding field of view on an adjacent, nondefective die. Both the single and the 1x2 array of defects are easily detected in this difference image.

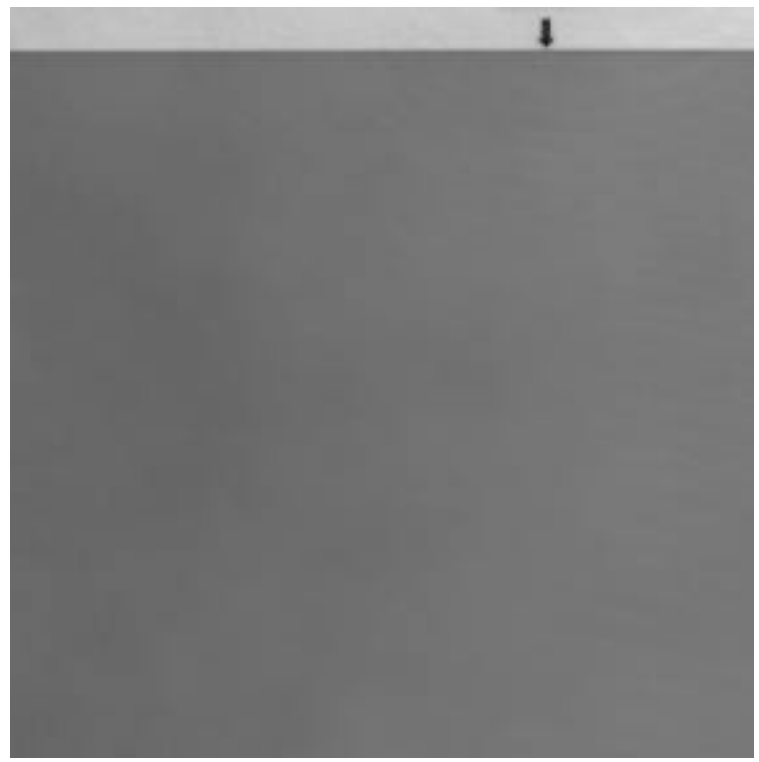

Figure 10. Phase image of a field of view taken at low magnification on the Fathom tool. The image includes both single and double under-etched contacts, which are not resolved, directly under the arrow visible at the top of the image. 


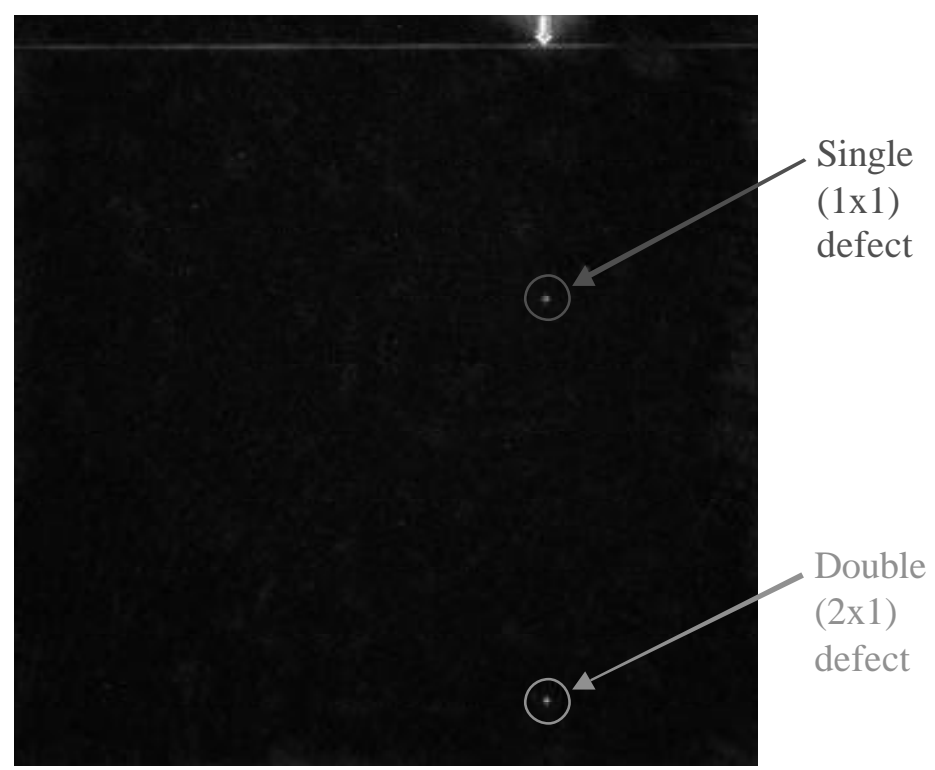

Figure 11. Difference image for the field of view shown in Figure 10. Both the single and double underetched contacts are clearly visible and easily detected.

\subsection{Visualization and detection of partial height extension defects}

As stated previously, both amplitude and phase differences can be used to detect defects. Amplitude-only difference images give the Fathom similar detection characteristics to current brightfield tools. Most of the defects detected by the Fathom tool that were identified as critical by fab engineers have had a stronger phase component than amplitude component. One such case is shown in Figure 12 below. The difference images were generated from a front-end etch layer. The particular defect is a partial height poly extension. The optical contrast between the defect and the background is small, resulting in no defect signal in the amplitude difference image (on the left in the figure). The phase difference image (on the right in the figure) shows the defect clearly. The height difference on the wafer causes a phase change that becomes visible upon differencing with a neighboring die. The same wafer was also scanned on a brightfield UV optical tool. Fathom caught an order of magnitude more partial height defects than did the amplitude only optical tool.
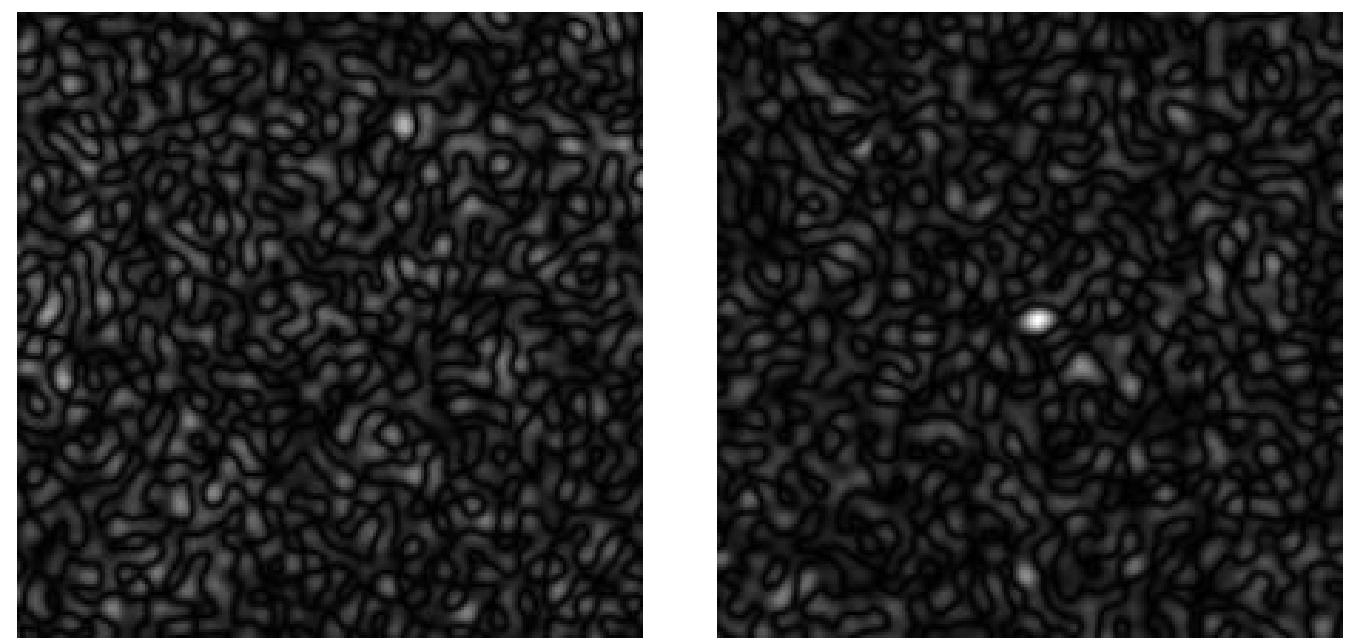

Figure 12. Difference images taken from a field of view with a partial height extension defect. (Left) Amplitude difference image. (Right) Phase difference image. The defect is clearly visible in the phase difference image but not in the amplitude difference image. 


\section{CONCLUSIONS}

We have shown case studies of a new digital holographic inspection technology that is able to find defects in high aspect ratio structures. Digital holography also provides phase information that allows us to visualize the topology of defects. Thus, the nLine Fathom tool has some unique defect detection capabilities not provided by existing inspection tool technologies.

\section{REFERENCES}

1. “2002 Update, International Technology Roadmap for Semiconductors," Semiconductor Industry Association, http://public.itrs.net/

2. E. N. Leith and J. Upatnieks, "Reconstructed wavefronts and communication theory," J. Opt. Soc. Amer., vol. 52, pp. 1123-1130, 1962.

3. E. N. Leith and J. Upatnieks, "Wavefront reconstruction with continuous-tone objects," J. Opt. Soc. Amer., vol. 53, pp. 1377-1381, 1963.

4. C. E. Thomas Jr., et al., "Direct to digital holography and holovision," U.S. Patent 6,078,392, June 20, 2000.

5. C. E. Thomas Jr. and G. R. Hanson, "Acquisition and replay systems for direct-to-digital holography and holovision," U.S. Patent 6,525,821, Feb. 25, 2003.

6. C. E. Thomas Jr., et al., "Direct to digital holography for semiconductor wafer defect detection and review," in A. Starikov and K. W. Tobin, eds., Design, Process Integration, and Characterization for Microelectronics, Proc. SPIE, vol. 4692, pp. 180-194, 2002.

7. C. E. Thomas Jr., et al., "Direct to digital holography for high aspect ratio inspection of semiconductor wafers." Proceedings of the 2003 International Conference on Characterization and Metrology for ULSI Technology, Austin, Texas, to appear, 2003.

8. E. Völkl, L. F. Allard, and B. Frost, "A software package for the processing and reconstruction of electron holograms," J. Microsc., vol. 180, Pt 1, pp. 39-50, 1995.

9. E. Voelkl, L. F. Allard, and B. Frost, "Electron holography: recent developments," Scanning Microsc., vol. 11, no. 1, pp. 405-414, 1996.

10. X. L. Dai, M. A. Hunt, and M. A. Schulze, "Automated image registration in the semiconductor industry: A case study in the direct to digital holography inspection system," Machine Vision Applications in Industrial Inspection XI, Proc. SPIE, vol. 5011, to appear, 2003

11. N. I. Fis her, Statistical analysis of circular data, Cambridge University Press, 1993.

12. E. Völkl, L. F. Allard, and D. C. Joy, Introduction to Electron Holography, Kluwer Academic/Plenum Publishers, 1999.

13. M Liebling, T. Blu, and E. Cuche, "A novel non-diffractive reconstruction method for digital holographic microscopy," Proceedings of the 2002 IEEE International Symposium on Biomedical Imaging, vol. 2, pp. 625-628, 2002.

14. U. Schnars and W. Juptner, "Direct phase determination in hologram interferometry with use of digitally recorded holograms," J. Opt. Soc. Am. A, vol. 11, pp. 2011-2015, 1994.

15. I. Yamaguchi and T. Zhang, "Phase-shifting digital holography," Opt. Lett., vol. 22, pp. 1268-1270, 1997.

16. R. J. Pryputniewicz, "Heterodyne holography applications in studies of small components," Opt. Eng., vol. 24, pp. 849-854, 1985.

17. C. Wagner, S. Seebacher, W. Osten, and W. Juptner, "Digital recording and numerical reconstruction of lensless Fourier holograms in optical metrology," Appl. Opt., vol. 38, pp. 4812-4820, 1999.

18. T.M. Kreis and W.P. Juptner, "Suppression of the de term in digital holography," Opt. Eng., vol. 36, pp. 2357-2360, 1997.

19. C. D. Depeursinge, et al., "Digital holography applied to microscopy," in S. Benton, et al., eds., Practical Holography XVI and Holographic Materials VIII, Proc. SPIE, vol. 4659, pp. 30-34, 2002.

20. I. Yamaguchi, et al., "Image formation in phase-shifting digital holography and applications to microscopy," Appl. Opt., vol. 40, no. 34, pp. 6177-6186, 2001. 
21. T. M. Kreis, M. Adams, and W. P. O. Jüptner, "Methods of digital holography: A comparison," in C. Gorecki, ed., Optical Inspection and Micromeasurements II, Proc. SPIE, vol. 3098, pp. 224-233, 1997.

22. J. H. Milgram and W. Li, "Computational reconstruction of images from holograms," Appl. Opt., vol. 41, no. 5, pp. 853-864, 2002.

23. L. Xu, et al., "Studies of digital microscopic holography with applications to microstructure testing," Appl. Opt., vol. 40, no. 28, pp. 5046-5051, 2001.

24. Y. Frauel, et al., "Distortion-tolerant three-dimensional object recognition with digital holography," Appl. Opt., vol. 40, no. 23, pp. 3887-3893, 2001.

25. O. Matoba, et al., "Real-time three-dimensional object reconstruction by use of a phase-encoded digital hologram," Appl. Opt., vol. 41, no. 29, pp. 6187-6192, 2002.

26. M. Tziraki, et al., "Photorefractive holography for imaging through turbid media using low coherence light," Appl. Phys. B, vol. 70, pp. 151-154, 2000.

27. F. Dubois, et al., "Border processing in digital holography by extension of the digital hologram and reduction of the higher spatial frequencies," Appl. Opt., vol. 41, no. 14, pp. 2621-2626, 2002.

28. J. H. Massig, "Digital off-axis holography with a synthetic aperture," Opt. Lett., vol. 27, no. 24, pp. 2179-2181, 2002.

29. R. B. Owen and A. A. Zozulya, "Comparative study with double-exposure digital holographic interferometry and a Shack-Hartmann sensor to characterize transparent materials," Appl. Opt., vol. 41, no. 28, pp. 5891-5895, 2002.

30. J. Khoury, et al., "Homodyne and heterodyne imaging through a scattering medium," Opt. Lett., vol. 26, no. 18, pp. 1433-1435, 2001. 